\title{
Persistent Pain: Physiotherapy Student Experiences of Person-Centred Care in Musculoskeletal Outpatient Departments
}

\author{
Aneurin Claydon \\ Cardiff and Vale University Health Board, United Kingdom \\ Graeme Paul-Taylor \\ Cardiff University, United Kingdom
}

\begin{abstract}
Person-centred care is championed throughout healthcare and emphasised in the management of persistent pain. Research reports that physiotherapists and physiotherapy students have struggled to integrate person-centred care into their practice and clinical work with people experiencing persistent pain. The aim of the study was to explore physiotherapy students' experiences of person-centred care with people with persistent pain in a musculoskeletal outpatients' placement setting, and to understand what affects physiotherapy students' ability to implement person-centred practice. An interpretive qualitative method was used with data being collected by semi-structured focus groups of a purposive sample of undergraduate final-year physiotherapy students. A thematic approach to data analysis was completed using a cyclical immersive process. Three themes emerged: understanding of person-centred care, learning, and influences on clinical practice. The students showed an understanding of person-centred care in line with current literature. Person-centred care was generally deemed 'well taught' at university however this did not translate into their practice in a placement setting. Several factors influenced their ability to implement person-centred care, and this impacted upon their learning and their management of people presenting with persistent pain. In conclusion, physiotherapy students struggled to integrate learning of person-centred care from university into their practice on a musculoskeletal outpatient's placement. It is recommended that physiotherapy course teams consider their curricula so that university and placement teaching facilitate person-centred learning and improve the management of persistent pain.
\end{abstract}

Keywords: persistent pain; person-centred care; physiotherapy; practice-based learning

${ }^{*}$ Corresponding Author: Aneurin Claydon, Cardiff and Vale UHB Headquarters, University Hospital of Wales (UHW), Heath Park, Cardiff CF14 4XW United Kingdom... Email: aneurin.claydon@wales.nhs.uk

Journal URL: http://e-learning.coventry.ac.uk/ojs/index.php/pblh

Claydon, A. and Paul-Taylor, G. (2017) 'Persistent pain: Physiotherapy student experiences of personcentred care in musculoskeletal outpatient departments'. International Journal of Practice-based Learning in Health and Social Care, 5 (2), 69-83

(c) 2017 Aneurin Claydon and Graeme Paul-Taylor. This Open Access article is distributed under the terms of the Creative Commons Attribution Attribution-Non-Commercial No Derivatives 4.0 International License (https://creativecommons.org/licenses/by-nc-nd/4.0/), which permits unrestricted noncommercial use, distribution, and reproduction in any medium, provided the original work is properly cited and is unaltered. 


\section{Introduction}

A person-centred health service has been a long-term goal for the National Health Service (NHS) in the United Kingdom (UK). Since 2000, an NHS that is centred on patients has been championed as part of healthcare reforms (Department of Health 2000), and in recent years the concept of person-centred care has surged in prominence, especially in the wake of the Mid Staffordshire report (Francis 2013) that investigated failings in care, and called for a culture change. Person-centred care forms a central tenet of clinical guidelines and healthcare policies (for example Bevan Commission 2015, Chartered Society of Physiotherapy 2017a, and National Institute for Health and Care Excellence [NICE] 2012). Elements of person-centred care form an integral part of the CSP's code of professional values (Chartered Society of Physiotherapy 2017a) and Quality Assurance standards (Chartered Society of Physiotherapy 2017b), highlighting its perceived importance in physiotherapy practice. Despite this increase in prominence, person-centred care remains a complex and broad paradigm.

A person-centred approach has been found to be particularly beneficial in the management of long-term conditions; it can play a significant role in improving symptoms, attitudes and behaviours, and quality of life (de Silva 2011). This study focused on persistent pain, where a person-centred approach to management has been championed by the Physiotherapy Pain Association (2014). It is a condition commonly seen by physiotherapists, especially in musculoskeletal (MSK) outpatient departments (OPD).

The Chief Medical Officer's report of 2008 stated that persistent pain affected around 7.8 million people in the UK (Donaldson 2009). Persistent pain is a unique and individual experience that can comprise of complex psychosocial factors which include the influence of beliefs and behaviours that can affect the person's experience of pain (Melzack 1999, Moseley 2003). It is known to have a profound impact on an individual's quality of life (Donaldson 2009). Approximately fifty per cent of individuals experiencing persistent pain report limitations undertaking social activities whilst twenty-five per cent become unemployed, and forty-nine per cent report experiences of depression (Donaldson 2009).

Studies have found that physiotherapists and physiotherapy students are aware of the importance of person-centred care and believe it to be beneficial (Mudge, Stretton, and Kayes 2014, Schmitt, Akroyd, and Akroyd 2012). However, it has been found that they have struggled to integrate a person-centred approach (Mudge, Stretton, and Kayes 2014), especially in the management of persistent pain (Sanders et al. 2013). Therefore, individuals with persistent pain may not be benefiting from best practice or achieving the outcomes that they could be. Concerns have been raised regarding the effectiveness of physiotherapists working with individuals with pain (Jones 2009). Furthermore, the potential struggle to maintain a personcentred approach as a practising physiotherapist may reflect the opportunities to develop these skills as a pre-registration student. The aim of this study was to explore the experiences of physiotherapy students being person-centred with people experiencing persistent pain in an MSK OPD placement setting, and expose the factors that may affect a student's ability to be person-centred.

\section{Methodology}

An interpretivist qualitative methodology was used to explore and uncover the experiences physiotherapy students had when on an MSK OPD placement (Neuman 2011). A purposive sample of final year physiotherapy students from a Higher Education Institution in the UK, with experience of managing persistent pain during one or more MSK outpatient department placements, was collected. An email invitation was sent to all final year students at the selected Institution via secure university email addresses. Nine students responded and were then emailed an information sheet, and were asked to confirm that they had the necessary experience required for the study. It was decided not to continue sampling to data saturation as 
it would be inappropriate to the study, as the aim was to uncover and understand the experiences of individual students.

A focus group, rather than interviews, was chosen for data collection as the group interaction can reveal attitudes and experiences from participants that would not be gained through other methods (Litosseliti 2003). Focus groups may better allow investigation into the meanings and interpretations of those participating and their decision-making processes (Barbour 2014). This is facilitated by the group interaction as a variety of perspectives can be gained and it allows a participant to review their own interpretations when faced with those of others, displaying how interpretations may be created or modified (Barbour 2007). A shared meaning can be developed by individual interpretations and identities drawing on a shared reality of being a physiotherapy student on an MSK OPD placement.

A semi-structured focus group was conducted in a quiet university room. Barbour (2007) states that three to eight participants in a focus group is sufficient as the more participants present, the more challenging moderation and analysis will be. Therefore, three of the nine students made up a pilot study and the other six composed another group (see Table 1). A topic guide was developed by the lead researcher, which was grounded on an extensive literature review and piloted. The topic guide consisted of four core questions with each question having several preprepared prompts to help aid discussion if necessary (see Table 2). Warm-up material was provided, which gave a definition and a background on persistent pain. The aim of this was to provide a shared definition of persistent pain and to give the participants an opportunity to reflect on their own experiences of persistent pain to facilitate the later discussion.

Table 1: Participants' demographics

\begin{tabular}{|l|l|l|l|l|l|l|l|l|l|l|l|}
\hline Participants & $\begin{array}{l}\text { Group } \\
\text { (pilot) }\end{array}$ & $\mathbf{1}$ & $\mathbf{2}$ & $\mathbf{3}$ & Group 2 & $\mathbf{4}$ & $\mathbf{5}$ & $\mathbf{6}$ & $\mathbf{7}$ & $\mathbf{8}$ & $\mathbf{9}$ \\
\hline Age & & 21 & 21 & 21 & & 20 & 20 & 21 & 21 & 21 & 21 \\
\hline Gender & & $\mathrm{F}$ & $\mathrm{M}$ & $\mathrm{M}$ & & $\mathrm{F}$ & $\mathrm{M}$ & $\mathrm{F}$ & $\mathrm{F}$ & $\mathrm{M}$ & $\mathrm{M}$ \\
\hline $\begin{array}{l}\text { Experience in an MSK } \\
\text { outpatient placement }\end{array}$ & & Yes & Yes & Yes & & Yes & Yes & Yes & Yes & Yes & Yes \\
\hline
\end{tabular}

Table 2: Example of question and prompts from topic guide

$$
\begin{aligned}
& \text { When seeing someone experiencing persistent pain in a musculoskeletal outpatient } \\
& \text { setting on placement, would you consider your practice to have been person centred? }
\end{aligned}
$$

- How do you think it was person centred?

- Why were you person-centred?

- Why don't you think you were person-centred? 
Moderation of the discussion was completed by the lead researcher whilst an assistant moderator was present to attempt to recognise the influence the lead researcher may have on data collection and interpretation to aid credibility. The assistant moderator made notes about the discussion and any points about the group dynamic or environment that were thought pertinent (Barbour 2007). The discussion was audio-recorded using a digital dictation device and hand-written field notes were completed by both the lead researcher and assistant moderator.

The data were transcribed using Express Scribe transcription software by the lead researcher who reviewed the audio files and transcripts. Data were used from the pilot study as it was thought to be transferable to the data gained from the main study, and there were only minimal modifications made to the topic guide between the two discussions. The lead researcher developed the themes with assistance from the assistant moderator and review by the research supervisor. The themes were derived from the data using Braun and Clarke's (2006) process of thematic analysis (see Table 3 ). This involved an iterative process of familiarisation with the data through immersing oneself in the data by multiple re-reading of the transcripts and relistening to the audio-files. Initial codes were then generated, where points of interest were taken from the text. This started the process of organising data in a meaningful way. Coding was completed manually by highlighting and noting parts of the text thought interesting, and matching recurring points. Themes were developed firstly by organising the codes into potential themes and sub-themes which were then reviewed leading to the naming of the final themes and sub-themes. Whilst the final definition of themes and sub-themes was completed by the lead researcher, triangulation was provided by the assistant moderator and review by the research supervisor. The participants were also offered the opportunity to review the transcripts and findings (although none of the participants responded). A reflexive diary was kept throughout the study process by the lead researcher to identify bias and to aim for transparency.

Table 3: Example of theme development

\begin{tabular}{|l|l|l|l|}
\hline Codes & Sub-theme & Theme & Evidence \\
\hline $\begin{array}{l}\text { We; working together; } \\
\text { shared decision-making; } \\
\text { goal-setting; onus on } \\
\text { them; relationship; } \\
\text { rapport; communication; } \\
\text { encouragement; talking; } \\
\text { respect; patient-comfort }\end{array}$ & Collaborative & $\begin{array}{l}\text { Understanding of } \\
\text { person-centred care }\end{array}$ & $\begin{array}{l}\text { '... We will have the } \\
\text { same goal and then we } \\
\text { are just finding a way } \\
\text { to suit the patient to do } \\
\text { it' (Ken). }\end{array}$ \\
\hline
\end{tabular}

Ethical approval was granted by the Cardiff University School of Healthcare Sciences Research Ethics Committee. Informed consent was gained prior to data collection, and a risk assessment completed. The participants created their own pseudonyms to use during the discussion.

Although the emergence of any sensitive issues was thought unlikely in the risk assessment, an assistant moderator was available to help if any situation became difficult (Barbour 2007). The contact details of the researcher and the research supervisor were provided in case any of the participants wished to discuss anything that occurred during the focus group or were upset with anything that had happened. Any raw data were saved onto a disc and submitted to the Higher Education Institution studied to be destroyed in accordance with their policy. 


\section{Results}

Figure 1: Themes of the study

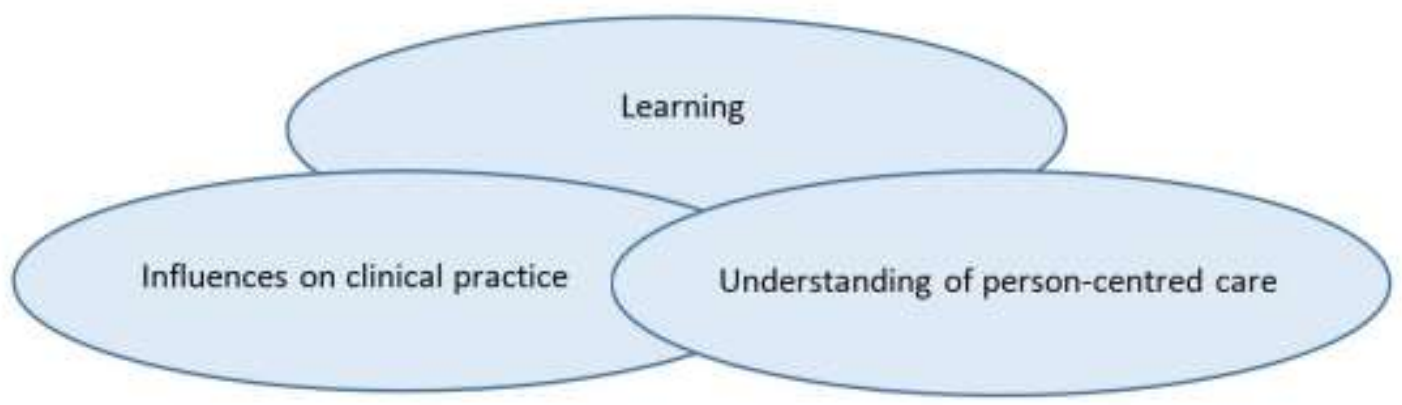

Theme 1: Understanding of person-centred care

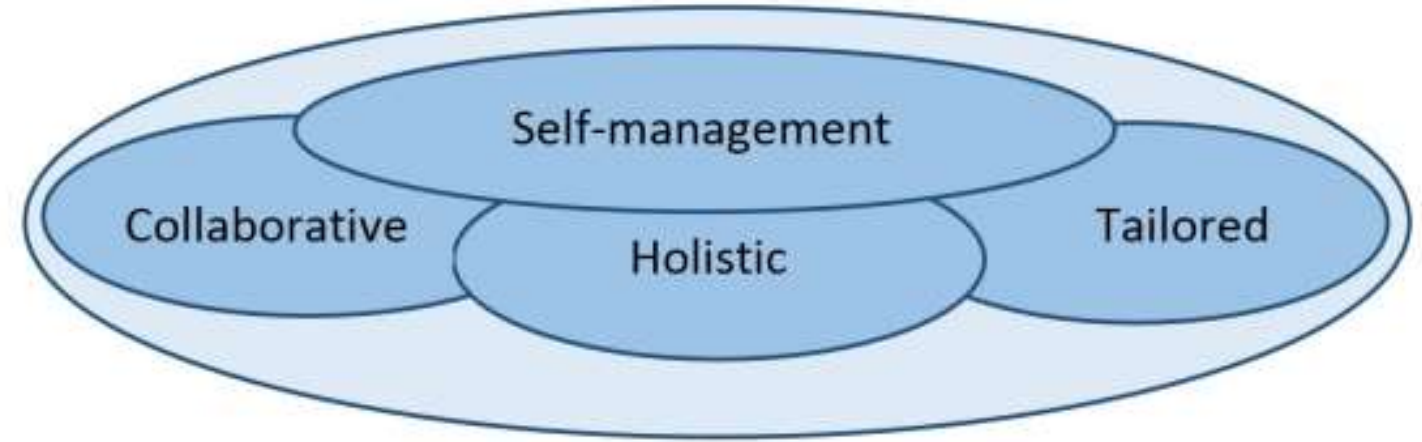

Figure 2 Subthemes of theme 'understanding of person-centred practice'

Four concepts of person-centred care arose from the discussion and were thought to be particularly relevant in the management of persistent pain. Developing a collaborative relationship was thought important as it encouraged the service-user to play a more active role.

My aim would be to have them as the most important person in the treatment room (Emily).

Being holistic was thought to be particularly pertinent, especially with the consideration of cognitive and psychosocial factors.

I think you got to take the whole picture like think holistically about it because you're not going to get to the bottom of it unless you treat that first (Jess).

Care also needed to be about 'tailoring your management to that patient' (Emily). Whilst a common idea was that 'you need to treat the person in front of you' (Emily). Tailoring seems to build on taking a holistic approach.

... understanding of the patient in terms of their working environment, their timetable ... and try to adapt the treatment and things like that (Ken).

By gaining a holistic understanding, it better allowed the students to tailor management (for example, around: age, lifestyle, and occupation) and that this should be a collaborative process. 
... Using the SMART [specific, measureable, agreed upon, realistic, time-based] goal principles to set out what he wanted to do and we worked through those (Tyrone).

Thus, these three components can help to develop an individual's self-management of their condition.

... Showing them and empowering them to be able to sort of manage their own symptoms because it's not always going to be a fix (Emily).

The end-goal of therapy was to move away from trying to cure an individual's persistent pain, and instead the emphasis centred on finding the best way to help the person manage their symptoms by themselves.

\section{Theme 2: Learning}

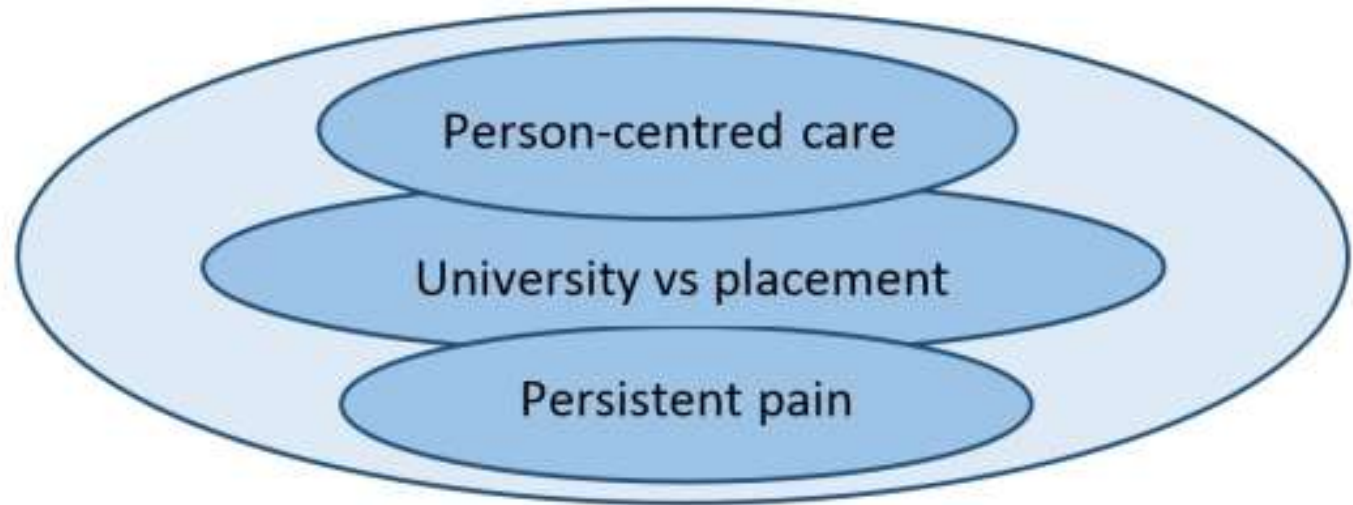

Figure 3 Subthemes of theme 'learning'

Overarching within this theme is a tension between the teaching experienced in the university and what is encountered when entering a clinical setting. At times, what was taught at university was at odds to what was learnt when on an MSK OPD placement.

It is drilled into us at university that it's really important to be patient-centred in our approach (Emily).

However, 'it's hard to apply it sometimes' (Geoff). Whilst being aware of the importance of taking a holistic and biopsychosocial approach from university, there seemed to be a gap in the students' skills to implement it. Especially, with people experiencing persistent pain 'it was too much of a minefield' (Lois), so they were encouraged to revert to a biomedical approach and thus some learnt to 'treat the problem rather than the patient' (Max).

The students highlighted gaps in their pain education; this included aspects of management such as medication and referral options whilst some believed that it was too theory-based and that they did not feel ready to manage the complexities of persistent pain.

It's all very well learning the theory behind all these sort of things, great, but when you actually see someone with it, it changes everything a little bit I feel (Tyrone).

Whereas students perceived that person-centred care in general was 'drilled into us' (Emily), they did not believe it was as comprehensively covered in the context of persistent pain.

We are definitely aware of person-centred care but I wouldn't say necessarily in the context of pain management (Max). 
Gaps in the students' knowledge of persistent pain were identified upon entering clinical realities and some students reported feeling that they lacked the skills to manage the complexities of persistent pain.

\section{Theme 3: Influences on clinical practice}

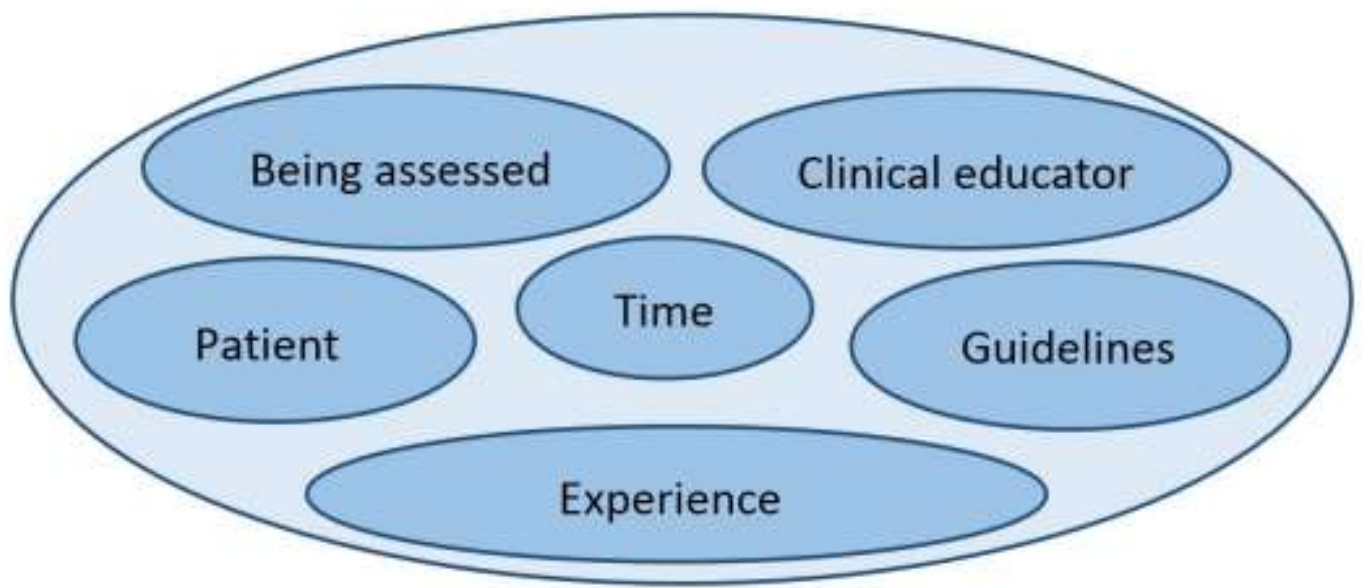

Figure 4 Subthemes of theme 'influences on clinical practice'

Several factors influenced the students' practice in managing persistent pain in an MSK OPD. Paramount were the pressure of being assessed and the influence of the clinical educator.

Yeah we're thinking about being patient-centred... but in the back of our heads we've all got that I kind of want to get seventy per cent mentality (Megan).

The students believed they needed to impress their clinical educator and adjust their practice to what they thought their clinical educator wanted as this might benefit their grade. This had a significant impact when dealing with persistent pain.

With chronic pain my clin ed's always been a bit like give them a few exercises there's nothing you can do for their chronic pain and tell them to go back to their GP (Max).

Time appeared to have an important aspect in the students' perceptions of what influenced their person-centred practice. Two main elements of time were mentioned: length of appointment and length of clinical placement.

You don't really have time to do anything but assess the pathology (Max).

I think four weeks for us as a student is a bit short (Geoff).

One more influence to be discussed is the 'patient'. This was related to the idea of people taking on 'patient roles' and not engaging with the physiotherapist.

Some people will come in then "right l've come and they're going to sort it out for me, they're going to get me moving, they're going to get me pain-free" (Geoff).

I think it depends on what kind of patient you have as well what their mentality was. I had a couple of blokes who definitely didn't want a student giving them advice on how to run their lives (Geoff). 
Some participants also felt that guidelines and protocol affected their practice, as they felt it led them to treat the problem rather than the individual.

Yeah and I think a lot of what limits person-centred care is protocol ... for some things you have to follow a protocol ... [it] limits person-centred care (Megan).

On a clinical placement, the students were more concerned with developing their clinical skills and becoming competent therapists rather than their ability to deliver a person-centred approach.

I think the more experience you have, as well, because when you're first on outpatients you're just so scared about ticking all the boxes (Jess).

However, they were optimistic that with greater experience, once their core skills have improved and they have become more efficient, there would be more opportunity to develop their personcentred practice.

\section{Discussion}

The physiotherapy students thought person-centred care was important and beneficial to their practice, as was also found by Schmitt, Akroyd and Akoyd (2012). Self-management was believed to be the goal of person-centred persistent pain management, and being holistic, collaborative and tailoring management appeared to be vital in developing this. The ideas of person-centred care described by the participants were in line with current literature (Collins 2014, Mead and Bower 2000, amongst others). Current research in pain shows that psychosocial factors can affect persistent pain and disability (Moseley 2007, O'Sullivan 2012) so a holistic approach is a necessity in its management (Hunter and Simmonds 2010). Tailoring care was also a collaborative process, and the students would attempt to find out what was important to the person and what they wanted to achieve. They would then work collaboratively to achieve the person's tailored goals. A mutually-beneficial relationship has been found to be a significant aspect of person-centred care (Mead and Bower 2000). Facilitating self-management was thought to be particularly relevant in persistent pain; rather than aiming to cure the pain, the students would aim to develop management of pain and enable the individual to carry on with their life.

Throughout the discussion there was an apparent dichotomy between what was learnt in university and what was learnt in practice, demonstrating a theory-practice gap. Despite finding a good understanding of person-centred care ('drilled into us' [Emily]), students struggled to implement it ('it's hard to apply it' [Geoff]). Due to the realities of being in an outpatients' department, and the complexities of managing persistent pain, they found translating their knowledge into practice a challenge. It is important for students to be able to develop generic and core skills, but they also need to be prepared to survive working in a real clinical setting (Hunt et al. 1998). Since the teaching of physiotherapy shifted to a more academic route into higher education and university, concerns have been raised regarding a deepening theorypractice gap (Roskell, Hewison, and Wildman 1998). Hunt, Adamson, and Harris (1998) found physiotherapy students believed there was a fundamental gap between the knowledge and skills taught at university and those needed in the clinical setting. Studies have shown that physiotherapists have struggled to incorporate person-centred care into practice ( $\underline{\mathrm{Cooper}}$, Smith, and Hancock 2009, Mudge, Stretton, and Kayes 2014).

The main aspect that the physiotherapists and physiotherapy students have found difficult is being biopsychosocial and holistic, feeling that they lack the skills to manage cognitive and psychosocial complexities and so revert to a biomedical paradigm (Etherton and Waterfield 2015, Sanders et al. 2013). Whilst Synnott et al. (2015) found that physiotherapists could identify cognitive and psychosocial issues in people with chronic low back pain, they also found that physiotherapists not only struggled to address these issues but stigmatised them. For conditions such as persistent pain - whereby cognitive and psychosocial components are 
commonly implicated - to be better managed, more must be done to develop the necessary skills in therapists to manage these issues. Studies have found changes to curriculum, including the introduction of narrative reasoning and psychosocial courses, improved person-centred and biopsychosocial attitudes and beliefs in physiotherapy students (Cruz, Caeiro, and Pereira 2014, Ross and Haidet 2011). Roskell, White, and Bonner (2013) found that a volunteered experiential placement helped to encouraged holistic, person-centred values in some physiotherapy students. However, this was not found in all students and did not have any longterm follow-up to see if these attitudes and values were maintained but shows potential for how changes in teaching could foster person-centeredness. Hunt, Adamson, and Harris (1998) highlighted the importance of educational review and how students are prepared for the workplace.

It has been found that, on average, physiotherapy students have a greater amount of pain education compared to other health disciplines (Briggs, Carrl, and Whittakerl 2011, WattWatson et al. 2009). These studies have not considered the quality of teaching though, with Etherton and Waterfield (2015) finding that physiotherapy students have felt unprepared to manage pain. Synnott et al. (2015) found physiotherapists believed that neither their undergraduate training nor professional development provided them with the competence to address the cognitive and psychosocial complexities in chronic low back pain. A change in physiotherapy curricula covering pain education has been recommended, using a collaborative, biopsychosocial and person-centred approach (International Association for the Study of Pain 2017, Jones and Hush 2011). It has also been found that pain neurophysiology education can be beneficial to physiotherapy students, improving knowledge and attitudes (Colleary et al. 2017). The university where the participants studied, employs an integrated approach to pain education whereas it is recommended that pain is taught independently from other modules of physiotherapy (International Association for the Study of Pain 2017). This could have led to the pain education received being diluted amongst other knowledge. However, pain education goes beyond university teaching. There should be an interaction between academic and clinical staff to allow better transition between the two areas, and this highlights the need for frequent and comprehensive review of pain education (Jones 2009).

Several factors were described that were implicated in the students' practice that influenced person-centred practice. Some of these related to the practicalities of being in a clinical setting; such as time pressures and having to follow protocols and guidelines. There was also the reality of seeing real 'patients'. In some cases, it appeared that the students were unprepared to deal with certain issues. Some 'patients' apparently saw it as the students' job to fix the problem and to do all the work as the individual adopted a 'patient role'. It has been found that people in pain can gain a 'patient identity' and desire a quick resolution from physiotherapy, and some physiotherapists admitted that they struggled to overcome these attitudes (Sanders et al. 2013). These students seemed to struggle to tackle this issue and motivate these 'patients'. This could make it difficult to form a collaborative relationship and facilitate self-management. Education and providing information to enable self-management was difficult with certain patients. Some students found it difficult to provide lifestyle advice and educate adults older than themselves, and found it difficult to motivate people who were not engaging. Sanders et al. (2013) also found a similar struggle in qualified physiotherapists, finding that they felt they lacked the necessary skills. Another concern was when someone was answering on the 'patient's' behalf (for example, when they were accompanied by a translator, partner or family member), there was a feeling that they were not treating the individual. Nonetheless, involving family members is thought to be an important in providing holistic and person-centred care (Pelzang 2010). One recommendation could be to provide students greater 'patient' experience and interaction prior to assessed clinical placements. One of the students suggested the possibility of more people who have been seen by physiotherapy attending lectures as guest speakers.

There was unanimous agreement amongst participants that outpatient appointments were not long enough. In a literature review, Pelzang (2010) identified time constraints as a barrier to person-centred care; reporting staff shortages and high demand on practitioners being significant contributing factors. This was also affected by the high demand, meaning 'get patient 
in, what's the problem, treat the problem, get them out, get the next patient in' (Max). They found it difficult to provide individualised care and build rapport, affecting their ability to be collaborative and holistic. In contrast, some students' experiences in quieter departments or community hospitals, where there was less pressure on waiting lists, felt they could take more time to talk through things and be more holistic. The students were seemingly not used to these kind of time pressures, and being on placement was there first experience of applying their knowledge and skills in these high-pressure situations. The students were optimistic that these aspects of their practice would improve with experience. Once they had improved their assessment and treatment skills and became more efficient, there would be more time to focus on being person-centred. When on placement, the focus was about learning and developing the basics, once that was done they could start to build on other facets of their practice. By moving away from routine practice and gaining clinical expertise, person-centred practice can develop (Petty, Scholes and Ellis 2011). The students appeared to believe that their development would follow a learning curve; with more time and greater experience, their skills and practice would improve and they can become more person-centred, starting to close the practice-theory gap.

Another element of time relates to the structure and format of clinical placements, as it was felt that the placement duration was not long enough. It did not allow them to see the whole experience of dealing with an individual with persistent pain; they felt they were only able to see a snapshot of their management. The students in this study believed time restrictions meant they struggled to individualise care, be holistic and develop a collaborative relationship as they may have only seen them once or twice. The structure of the course at the university studied, consists of a greater number of shorter length placements compared to fewer placements that last longer. Whilst this allows greater breadth and provides a more diverse learning experience, some students felt they had missed out on learning opportunities and that some learning experiences were unfulfilled.

The other two factors also relate to the placement as a learning and teaching experience: being assessed, and the clinical educator. Getting a good mark on their placement was important to the students and had an ambivalent effect on aspects of their person-centred practice. Some students commented that they might improve their mark by demonstrating, for example, that they individualised the care they provided. However, most said that there was pressure to be efficient, and that over-running sessions might negatively affect their mark. Therefore, they felt that they were not able to 'talk to them about everything else that is impacting other stuff (Emily). So rather than taking the time to develop a relationship with the individual and get a whole picture, they felt they just had to 'get patient in, what's the problem, treat the problem, get them out, get the next patient in' (Max). Consequently, the motivation of getting a good grade could overcome the motivation to be person-centred. The clinical educator has the power to decide marks and the students described a need to be 'clin-ed centred' (Ken). In one of the participant's experiences, his clinical educator had a negative attitude towards people with persistent pain. Lindquist et al. (2006) found that clinical educators are important role-models to students and can influence how physiotherapy students identify themselves, including whether they see themselves as person-centred. This participant felt he needed to treat people with persistent pain as his clinical educator would, otherwise it might have negative consequences. Clinical educators hold power over the student; by sharing negative attitudes towards persistent pain, the students might develop the same beliefs since they might receive a better grade whilst performing this practice. Therefore, a need to be 'clin-ed centred' overshadows the desire to be person-centred. Clinicians without expertise in pain may not be best placed to develop a student's learning (Jones 2009). On the other hand, other students reported clinical educators who were more encouraging of person-centred practice and who had more positive attitudes towards persistent pain, and this could reinforce person-centred behaviours. Physiotherapy education may be too teacher-focused, and moving away from the 'clin-ed centred'-ness described in this study to a more student-centred way of teaching may facilitate personcenteredness (Petty 2009). 


\section{Limitations}

One of the limitations of the study was that the lead researcher (who also moderated the focus groups and analysed the data) was a member of the student group that was being studied. Within the reflexive diary, the researcher identified that bias could arise from this as they would have had their own experiences and would have their own ideas and assumptions. Participants can respond to prejudices by providing information influenced by the researcher that may not reflect the participant's true feelings, and this might affect the credibility of the present study (Litosseliti 2003). Conversely, the researcher also reflected that having had similar experiences to the participants could have meant that they had a greater understanding of the experiences uncovered. Also, the participants would also be previously acquainted with the researcher, and while on the one hand this may make them more comfortable, it may influence the information they provide (Litosseliti 2003). All the participants declined the opportunity to provide respondent validation. However an assistant moderator, who had no other affiliation with the study, was present to provide accuracy and triangulation. The assistant moderator did complete field notes about the interactions within the discussion and no comments were made regarding any interaction between any of the participants and the moderator. This study looked at a small number of students (from a single University); and rather than seeking saturation, it fulfilled its aims of exploring and capturing a group of individual students' experiences.

\section{Conclusion}

Physiotherapy students struggled to integrate knowledge of person-centred care into their clinical practice on an MSK OPD placement. Despite feeling person-centred care was well taught at university, what they had learnt appeared to be at odds with the realities of clinical practice. A range of factors - seemingly contributing to these students' ability to be personcentred - have been exposed. Pain education was thought to be inadequate, with little emphasis on a person-centred approach and not preparing students to manage the complexities and realities of persistent pain in a clinical setting. University physiotherapy courses should aim to encourage person-centred practice in physiotherapy students as future practitioners. Future research into this topic could further investigate the influences upon physiotherapy students who are learning how to include person-centred care into their practice and how to enable the development of best practice. The exploration of physiotherapy students' perceptions of pain education may help inform how to integrate person-centred care into their practice. Universities could review their curriculum to facilitate a conducive relationship between university-based and practice-based learning, to foster person-centred development. Stemming from the findings, possible areas that could be reviewed are the duration of placements and how clinical placements are assessed. Another area that could be explored is how wellprepared clinical educators are to address the issues raised from the study, and whether there needs to be greater awareness of the struggles facing students when assimilating into a placement setting from university within this context.

\section{Acknowledgements}

We would like to thank Cardiff University School of Healthcare Sciences for the facilities and equipment used for the study, and Cardiff University Physiotherapy department for the support provided. We also wish to thank all of the students who participated in the study. 


\section{References}

Barbour, R. (2007) Doing focus groups. London: Sage https://doi.org/10.4135/9781849208956

Barbour, R. (2014). Introducing qualitative research: a student's guide. 2nd edn. London: Sage

Bevan Commission (2015) A Prudent Approach To Health: Prudent Health Principles. [online]. available from http://www.bevancommission.org/prudent-healthcare [6 November 2017]

Braun, V., and Clarke, V. (2006) 'Using thematic analysis in psychology'. Qualitative Research in Psychology, 3 (2), 77-101 https://doi.org/10.1191/1478088706qp063oa

Briggs, E.V., Carrl, E.C.J., and Whittakerl, M.S. (2011) 'Survey of undergraduate pain curricula in healthcare professionals in the United Kingdom'. European Journal of Pain, 15, 789795. https://doi.org/10.1016/j.ejpain.2011.01.006

Chartered Society of Physiotherapy. (2017a) Code of Members' Professional Values and Behaviour'. [online]. available from <http://www.csp.org.uk/publications/code-membersprofessional-values-behaviour> [30 October 2017]

Chartered Society of Physiotherapy (2017b) Quality Assurance Standards for Physiotherapy Service Delivery. [online]. available from <http://www.csp.org.uk/publications/qualityassurance-standards $>$ [30 October 2017]

Colleary, G., O'Sullivan, K., Griffin, D., .Ryan, C.G., and Martin, D.J. (2017). 'Effect of pain neurophysiology education on physiotherapy students' understanding of chronic pain, clinical recommendations and attitudes towards people with chronic pain: A randomised controlled trial'. Physiotherapy. [preprint] http://dx.doi.org/10.1016/i.physio.2017.01.006

Collins, A. (2014) Measuring What Really Matters: Towards a Coherent Measurement System to Support Person-Centred Care. Health Foundation, Thought Paper. [online]. available from <http://www.health.org.uk/sites/health/files/MeasuringWhatReallyMatters.pdf> [15 March 2017]

Cooper, K., Smith, B.H., and Hancock, E. (2009) 'Patients' perceptions of self-management of chronic low back pain: Evidence for enhancing patient education and support'. Physiotherapy, 95 (1) 43-50. https://doi.org/10.1016/i.physio.2008.08.005

Cruz, E.B., Caeiro, C., and Pereira, C. (2014) 'A narrative reasoning course to promote patientcentred practice in a physiotherapy undergraduate programme: A qualitative study of final year students'. Physiotherapy Theory and Practice, 30 (4), 254-260. http://dx.doi.org/10.3109/09593985.2013.863415

de Silva, D. (2011) 'Helping People Help Themselves: A Review of the Evidence Considering Whether it is Worthwhile to Support Self-Management'. Health Foundation Evidence Report. [online]. available from <http://www.health.org.uk/publication/evidence-helpingpeople-help-themselves> [15 March 2017]

Department of Health (2000) The NHS Plan: A plan for Investment, A Plan for Reform. [online]. available from <http://webarchive.nationalarchives.gov.uk/20130107105354/http://www.dh.gov.uk/prod consum dh/groups/dh digitalassets/@dh/@en/@ps/documents/digitalasset/dh 11852 2.pdf> [15 March 2017 ]

Donaldson, L. (2009). 150 Years of the Annual Report of the Chief Medical Officer (2008). [online]. available from 
<http://webarchive.nationalarchives.gov.uk/20130107105354/http:/www.dh.gov.uk/prod consum dh/groups/dh digitalassets/documents/digitalasset/dh 096231.pdf > [15 March 2017]

Etherton, J. and Waterfield, J. (2015) 'Preparing to manage patients in pain. The student perspective: A pilot'. Pain and Rehabilitation - the Journal of Physiotherapy Pain Association, 38, 27-33

Francis, R. (2013) Report of the Mid Staffordshire NHS Foundation Trust Public Inquiry. London: The Stationery Office [online]. available from $<$ http://webarchive.nationalarchives.gov.uk/20150407084003/http://www.midstaffspublic inquiry.com/report> [19 March 2017]

Hunt, A., Adamson, B., and Harris, L. (1998) 'Physiotherapists' perceptions of the gap between education and practice'. Physiotherapy Theory and Practice, 14 (3), 125-138 http://dx.doi.org/10.3109/09593989809057157

Hunt, A., Adamson, B., Higgs, J. and Harris, L. (1998) 'University education and the physiotherapy professional'. Physiotherapy 84 (6), 264-273 https://doi.org/10.1016/S0031-9406(05)65527-7

Hunter, J.P. and Simmonds, M.J. (2010) 'Pain: Putting the whole person at the centre'. Physiotherapy Canada, 62 (1). 1-4. https://doi.org/10.3138/physio.62.1.1

International Association for the Study of Pain (2017) 'IASP Curriculum Outline on Pain for Physical Therapy'. [online]. available from $<$ http://www.iasppain.org/Education/CurriculumDetail.aspx?ltemNumber=2055> [15 March 2017]

Jones, L. (2009) 'Implications of IASP core curriculum for pre-registration physiotherapy education'. British Journal of Pain, 3 (1), 11-15 https://doi.org/10.1177/204946370900300104

Jones, L. and Hush, J.M. (2011) 'Pain education for physiotherapists: Is it time for curriculum reform?' Journal of Physiotherapy [online]. 57 (4), 207-208. available from $<$ http://www.journalofphysiotherapy.com/article/S1836-9553(11)70049-2/abstract> [2 July 2017]

Lindquist, I., Engardt, M., Garnham, L., Poland, F., and Richardson, B. (2006) 'Physiotherapy students' professional identity on the edge of working life'. Medical Teacher, 28 (3), 270-276. http://dx.doi.org/10.1080/01421590600605272

Litosseliti, L. (2003) Using Focus Groups in Research. London: Continuum.

Mead, N. and Bower, P. (2000) 'Patient-centeredness: A conceptual framework and review of the empirical literature'. Social Science \& Medicine, 51 (7), 1087-1110 https://doi.org/10.1016/S0277-9536(00)00098-8

Melzack, R. (1999) 'From the gate to the neuromatrix'. Pain [online]. 82 (supplement 1), S121S126. available from <http://www.sciencedirect.com/science/article/pii/S0304395999001451> [2 July 2017]

Moseley, G.L. (2003) 'A pain neuromatrix approach to patients with chronic pain'. Manual Therapy, 8 (3), 130-140 https://doi.org/10.1016/S1356-689X(03)00051-1

Moseley, G.L. (2007) 'Reconceptualising pain according to modern pain science' Physical Therapy Reviews, 12, 169-178. http://dx.doi.org/10.1179/108331907X223010 
Mudge, S., Stretton, C., and Kayes, N. (2014) 'Are physiotherapists comfortable with personcentred practice? An autoethnographic insight'. Disability and Rehabilitation, 36 (6), 457-463. http://dx.doi.org/10.3109/09638288.2013.797515

National Institute for Health and Care Excellence (NICE) (2012) 'Patient Experience in Adult NHS Services'. [online]. available from <https://www.nice.org.uk/guidance/qs15> [15 March 2017]

Neuman, W.L. (2011) Social Research Methods: Qualitative and Quantitative Approaches. 7th edn. London: Pearson.

O'Sullivan, P. (2012) 'It's time for change with the management of non-specific chronic low back pain'. British Journal of Sports Medicine, 46, 224-227. http://dx.doi.org/10.1136/bjsm.2010.081638

Pelzang, R. (2010) 'Time to learn: Understanding patient-centred care'. British Journal of Nursing, 19 (14), 912-917. https://doi.org/10.12968/bjon.2010.19.14.49050

Petty, N.J. (2009) 'Towards clinical expertise: learning transitions of neuromusculoskeletal physiotherapists'. [online]. Doctoral thesis. University of Brighton. available from<http://eprints.brighton.ac.uk/14076/> [19 March 2017]

Petty, N.J., Scholes, J., and Ellis, L. (2011) 'Master's level study: Learning transitions towards clinical expertise in physiotherapy'. Physiotherapy, 97 (3), 218-225.

https://doi.org/10.1016/i.physio.2010.11.002

Physiotherapy Pain Association (2014) 'Physiotherapy framework - Entry level graduate to Expert: Describing the Values, Behaviours, Knowledge and Skills of Physiotherapists Working with People in Pain'. [online]. available from $<$ http://ppa.csp.org.uk/documents/ppa-physiotherapy-framework-entry-level-graduateexpert-describing-values-behaviours> [15 March 2017]

Roskell, C., Hewison, A., and Wildman, S. (1998) 'The theory-practice gap and physiotherapy in the UK: Insights from the nursing experience'. Physiotherapy Theory and Practice, 14 (4), 223-233. http://dx.doi.org/10.3109/09593989809057168

Roskell, C., White, D. and Bonner, C. (2013) 'Developing patient-centred care in health professionals: reflections on introducing service-learning into the curriculum'. International Journal of Therapy and Rehabilitation, 19 (8), 448-456. https://doi.org/10.12968/ijtr.2012.19.8.448

Ross, E.F. and Haidet, P. (2011) 'Attitudes of physical therapy students toward patient-centered care, before and after a course in psychosocial aspects of care'. Patient Education and Counseling, 85 (30), 529-532. https://doi.org/10.1016/j.pec.2011.01.034

Sanders, T., Foster, N.E., Bishop, A., and Ong, B.N. (2013) 'Biopsychosocial care and the physiotherapy encounter: Physiotherapists' accounts of back pain consultations'. BMC Musculoskeletal Disorders, 14 (65). https://doi.org/10.1186/1471-2474-14-65

Schmitt, J., Akroyd, K., and Akroyd, L. (2012) 'Perceptions of physiotherapy students of a person-centred approach in rehabilitation'. International Journal of Therapy and Rehabilitation 19 (1), 23-30. https://doi.org/10.12968/IJTR.2012.19.1.23

Synnott, A., O'Keeffe, M., Bunzli, S., Dankaerts, W., O'Sullivan, P., \& O'Sullivan, K. (2015) 'Physiotherapists may stigmatise or feel unprepared to treat people with low back pain 
and psychosocial factors that influence recovery: A systematic review'. Journal of Physiotherapy, 61 (2), 68-76. https://doi.org/10.1016/j.jphys.2015.02.016

Watt-Watson, J., McGillion, M., Hunter, J., Choiniere, M., Clark, A.J., Dewar, A., . . Webber, K. (2009) 'A survey of prelicensure pain curricula in health science faculties in Canadian universities'. Pain Research and Management, 14, 439-444

https://doi.org/10.1155/2009/307932 